Perhaps the newly detected substance is a prime means of food seeking (it is usually abundant during the vegetative stage when bacteria are present), whereais cyclic-AMP acts specifically in the aggregation process. The identity of the new substance will be keenly awaited. Its non-diffusibility does not especially worry Bonner and his colleagues; such a feature may have little significance in nature where soil and humus are the normal substrates.

\section{TRANSFORM FAULTS A Job for Coriolis}

\section{from our Geomagnetism Correspondent}

Is spite of all that has been written about mid-oceanic ridges in general, and about transform faults in particular, no one seems to have given a really satisfactory explanation of why the faults are there. In his original description of transform faults, Tuzo Wilson supposed that the offsets were inherited from the shape of the break which first formed between the coasts of the adjacent continents-but really that just begs the question. And the question is quite important because, as Howell points out (J. Geophys. Res., 75, 2769; 1970), it is difficult to reconcile the offsets with a smoothly flowing convective system in the mantle. The sea floor spreads normally from each ridge section, but because each section lies at a small angle to the overall trend of the ridge the upwelling crest of any convective flow must be discontinuous.

Howell has come up with an ingenious explanation of how the bulk convective flow can be continuous and yet still be consistent with offsets. He suggests that it is all due to the Coriolis force, which causes the surface of the convection cell to drift sideways relative to the underlying flow. There is a well established precedent for such a phenomenon in the circulation of oceans and atmospheres. In the oceans, for example, a current at one level produces a flow at an adjoining level which is rotated clockwise in the northern hemisphere and anticlockwise in the southern hemisphere. In this case the effect can be so great that the net flow is normal to the surface driving force.

An obvious way to test Howell's hypothesis is to determine the sense of apparent rotation of the ridge sections relative to the overall trend. It turns out that more than two-thirds of the displacements are in the sense required by the Coriolis effect. Although this is a large enough difference to support Howell's idea, it suggests that Coriolis force is not the whole explanation. A second test derives from the variation of Coriolis force with latitude. Because the force is proportional to the sine of latitude, fault displacements should tend to be larger at high latitudes than near the equator. According to Howell, the total displacement along transform faults between $0^{\circ}$ and $30^{\circ}$ latitude is $61 \cdot 4$ arbitrary units, compared with 72.5 such units for faults between $30^{\circ}$ and $60^{\circ}$. Unfortunately there are too few data above $60^{\circ}$ to enable the comparison to be extended.

Hitherto, little attention has been given to the effect of the Coriolis force on mantle convection because for slow movements it is small. Runcorn, for example (J. Geophys. Res., 69, 4389; 1964), estimated that the Coriolis force was only $10^{-8}$ as large as the other forces involved. But the limits of error on this estimate are certainly several orders of magnitude because neither the size of the convection cells nor the viscosity of the mantle maierial was known. Furthermore, there is no simple relationship between the magnitude of the forces and the amount of rotation they produce. Much depends on the coupling between the driving force and the mantle material and the thickness and viscosity of the layers through which the rotation is distributed. For these reasons it is impossible to give a realistic quantitative estimate of the effect of Coriolis force and thus definitely to rule it out. On the contrary, Howell shows experimentally that the Coriolis force is involved in some way. But because of its small size it is not surprising that it is not completely dominant.

\section{AMORPHOUS SOLIDS Shapely Currents in Carbon}

\section{by our Solid State Physics Correspondent}

Electrical conduction in amorphous carbon seems to occur by a rich variety of processes, according to experiments by C. J. Adkins, S. M. Freake and E. M. Hamilton on thin films of carbon held in different conditions (Phil. Mag., 22, 183; 1970). At low applied fields, conduction along the films was found to be by a hopping process characterized by a special temperature variation with voltage, whereas at higher fields the mechanism was of the Poole-Frenkel type with a range of traps and a large effective dielectric constant. Conduction through the film was found to be appreciably poorer than along it, which was attributed to structural anisotropy in the films.

Within the past two or three years there has been a considerable upsurge of interest in the electrical properties of amorphous materials, and a recent review by a panel of the Science Research Council gave every indication that this interest will continue to flourish (Nature, 22\%, 108; 1970). Carbon is a particularly suitable material to study in thin film form as the amorphous character of these films is strong. Electron microscope examinations suggest that the ordered structure is limited to $30 \AA$ or less and that the crystallite size is typically no more than $10 \AA$.

Extremely large variations of resistance were found between different specimens. supposedly caused by variations in grain size and impurity content. The dependence of the resistance on temperature, however, at low applied fields and low temperatures was the same for each specimen, and followed the pattern predicted by Mott in which there is sufficient overlap of the localized states for tunnelling to occur beyond the nearest localized site.

At higher fields, the variation of current with applied ficld and temperature suggested to Adkins et al. the presence of Poole-Frenkel conduction. The parameter which denotes the trap depth turned out to be quite well defined above about $10 \mathrm{~K}$, but decreased rapidly at lower temperatures. They point out that the drop in trap depth is not surprising as the extreme disorder implies a spectrum of depths of which only the most shallow would remain active at low temperatures.

They found that conduction through the film at low fields defied any simple explanation. The chief surprise was the flattening off of the resistance at $5 \mathrm{~K}$ as the temperature was lowered. 